\title{
Phase II study of neoadjuvant chemotherapy with irinotecan hydrochloride and nedaplatin followed by radical hysterectomy for bulky stage Ib2 to IIb, cervical squamous cell carcinoma: Japanese Gynecologic Oncology Group study (JGOG 1065)
}

\author{
SATOSHI YAMAGUCHI ${ }^{1}$, RYUICHIRO NISHIMURA ${ }^{1}$, NOBUO YAEGASHI $^{2}$, KAZUSHIGE KIGUCHI $^{3}$, \\ TORU SUGIYAMA ${ }^{4}$, TSUNEKAZU KITA ${ }^{5}$, KANEYUKI KUBUSHIRO ${ }^{6}$, KATSUJI KOKAWA ${ }^{7}$, \\ MASAMICHI HIURA $^{8}$, KATSUMI MIZUTANI ${ }^{9}$, KAICHIRO YAMAMOTO ${ }^{10}$ and KEN TAKIZAWA $^{11}$ \\ ${ }^{1}$ Department of Gynecologic Oncology, Hyogo Cancer Center, 13-70 Kitaoji-cho, Akashi, Hyogo 673-8558; \\ ${ }^{2}$ Department of Obstetrics and Gynecology, Tohoku University School of Medicine Hospital, 1-1 Seiryou-cho, \\ Aoba-ku, Sendai, Miyagi 980-8574; ${ }^{3}$ Department of Obstetrics and Gynecology, St. Marianna University School \\ of Medicine Hospital, 2-16-1 Sugao, Miyamae-ku, Kawasaki, Kanagawa 216-8511; ${ }^{4}$ Department of Obstetrics and \\ Gynecology, Iwate Medical University Hospital, 19-1 Uchimaru, Morioka, Iwate 020-8505; ${ }^{5}$ Department of Obstetrics \\ and Gynecology, Nara Hospital, 30-1 Hiramatsu, Nara 631-0846; ${ }^{6}$ Department of Obstetrics and Gynecology, \\ Toho University Ohashi Hospital, 2-17-6 Ohashi, Meguro-ku, Tokyo 153-8515; ${ }^{7}$ Kokawa Clinic, 366-4 Musotani, \\ Wakayama 640-8482; ${ }^{8}$ Department of Gynecology, Shikoku Cancer Center, 160 Kou, Minamiumemoto-cho, \\ Matsuyama, Ehime 791-0280; ${ }^{9}$ Tokyo Metropolitan Cancer and Infectious Diseases Center Komagome Hospital, \\ 3-18-22 Honkomagome, Bunkyo-ku, Tokyo 113-8677; ${ }^{10}$ Department of Obstetrics and Gynecology, \\ Sakai Hospital Kinki University School of Medicine, 2-7-1 Harayamadai, Mimami-ku, Sakai, \\ Osaka 590-0132; ${ }^{11}$ Department of Gynecology, Cancer Institute Hospital, \\ Ariake, 3-10-6 Ariake, Koto-ku, Tokyo 135-8550, Japan
}

Received January 24, 2012; Accepted April 3, 2012

DOI: 10.3892/or.2012.1814

\begin{abstract}
The efficacy and adverse events of neoadjuvant chemotherapy with irinotecan hydrochloride and nedaplatin were evaluated in patients with bulky stage Ib2 to IIb cervical squamous cell carcinoma. Eligibility included patients who received irinotecan $\left(60 \mathrm{mg} / \mathrm{m}^{2}\right)$ on days 1 and 8 and nedaplatin $\left(80 \mathrm{mg} / \mathrm{m}^{2}\right)$ on day 1 of a 21 -day cycle. After 1-3 courses of chemotherapy, radical hysterectomy was performed. Sixty-eight patients were enrolled. Sixty-six were included in the full analysis set. Their median age was 47 years (range 22-71), the FIGO stage was Ib2 in 18 patients, IIa in 10 , and IIb in 38. Radical hysterectomy was performed after NAC in 63 patients $(95.5 \%)$. The number of administered courses of NAC was 1 in 13 patients, 2 in 43, and 3 in 10 . The response rate, the primary endpoint of this study,
\end{abstract}

Correspondence to: Dr Satoshi Yamaguchi, Department of Gynecologic Oncology, Hyogo Cancer Center, 13-70 Kitaoji-cho, Akashi, Hyogo 673-8558, Japan

E-mail: s-yama@hp.pref.hyogp.jp

Key words: irinotecan hydrochloride, nedaplatin, neoadjuvant chemotherapy, cervical cancer, squamous cell carcinoma, JGOG1065 was $75.8 \%$ (CR in 2 patients, $\mathrm{PR}$ in $48, \mathrm{SD}$ in 12 , $\mathrm{PD}$ in 0 , and $\mathrm{NE}$ in 4). The mean number of treatment courses required for a response was 1.42 ( 1 course in 30 patients, 2 courses in 19, and 3 courses in 1). The incidences of grade 3 or 4 hematological toxicities were: neutropenia $72.2 \%$, leukopenia $16.7 \%$, anemia $13.6 \%$, thrombocytopenia $7.6 \%$, febrile neutropenia $1.5 \%$, and elevations of alanine aminotransferase and aspartate aminotransferase $1.5 \%$. Grade 3 or 4 non-hematologic toxicities were as follows: diarrhea $6.1 \%$, nausea $3 \%$, anorexia $1.5 \%$, vomiting $1.5 \%$, fever $1.5 \%$, allergic reactions $1.5 \%$, ileus $1.5 \%$ and vesicovaginal fistula $1.5 \%$. Neoadjuvant chemotherapy with irinotecan and nedaplatin was an effective and well-tolerated treatment for patients with bulky stage Ib2 to IIb squamous cell carcinoma of the uterine cervix.

\section{Introduction}

We previously reported a phase I study of combination chemotherapy with irinotecan hydrochloride (CPT-11) and nedaplatin (NED) for cervical squamous cell carcinoma (JGOG 1063) (1). We conducted a phase II clinical trial to evaluate the effectiveness and toxicity of neoadjuvant chemotherapy (NAC) with CPT-11 and NED in women with stage Ib2 or II cervical squamous cell carcinoma. 
CPT-11 is a DNA topoisomerase I inhibitor developed in Japan. In a phase II study in patients with cervical carcinoma, it exhibited relatively high efficacy, with a response rate of $23 \%$ (2).

NED is a second-generation platinum compound developed in Japan. The response rate was $46 \%$ to patients with cervical cancer in phase II clinical trials. Its antitumor activity was suggested to be at least equivalent to that of cisplatin (CDDP). Since NED is less nephrotoxic than CDDP, its indication range was extended to patients with renal dysfunction. NED can be administered on an outpatient basis, without rehydration therapy. In vitro studies using gynecological cancer cell lines NDP exerted stronger antitumor activity than CDDP (3).

Patients with cervical cancer have been reported to have a high rate of response to CPT-11 plus CDDP (4-7). To further enhance efficacy and safety, CPT-11 has been combined with nedaplatin. Good outcomes have been reported (8-10). Clinically, Ohwada et al reported a high response rate of $81 \%$ in patients with primary cervical cancer (11). Combined therapy with CPT-11 and NED is thus expected to be useful for the management of advanced cervical cancer.

The purpose of NAC in this study was to increase the radicality of surgery by inducing tumor shrinkage, not to achieve a histological complete response. Therefore, patients could receive up to 3 courses of NAC, but if PR or CR was achieved during 1 or 2 courses, the physician could decide whether to proceed to surgery. NAC regimens need a high local response rate of the primary tumor and a prompt onset of effect.

\section{Patients and methods}

The study group comprised women in whom radical hysterectomy was indicated, but surgery was considered difficult. In clinical practice, this category includes patients with bulky tumors and those with high-grade parametrial invasion. Clinical stage Ib2 and IIa tumors were defined as measurable lesions $>4 \mathrm{~cm}$ in diameter, and clinical stage IIb tumors were defined as measurable lesions $>2 \mathrm{~cm}$ in diameter. Patients were enrolled from January 2007 through July 2007 at member hospitals of the Japanese Gynecologic Oncology Group (JGOG). The study protocol was approved by the institutional review board of each participating hospital. All patients provided informed consent before enrollment.

Eligibility criteria. Eligibility criteria were as follows: i) a histopathologically confirmed diagnosis of cervical cancer (squamous cell carcinoma); ii) any of the following clinical stages according to the Federation of Gynecology and Obstetrics (FIGO) staging system (1994 version): stage Ib2, stage IIa [measurable lesions $>4 \mathrm{~cm}$ in greatest diameter on direct measurement or magnetic resonance imaging (MRI)], or stage $\mathrm{IIb}$ (measurable lesions $>2 \mathrm{~cm}$ in greatest diameter on direct measurement or MRI); iii) the primary tumor can be directly measured or measured on MRI; iv) no previous treatment; v) an age of 20-75 years at enrollment; vi) a performance status (Eastern Cooperative Oncology Group) of 0 or 1; vii) extended hysterectomy is feasible; viii) preserved function of major organs (bone marrow, heart, liver, kidney, etc.) [neutrophil count $\geq 2000 / \mu 1$, platelet count $\geq 100 \times 10^{3} / \mu 1$, hemoglobin level $\geq 9.0 \mathrm{~g} / \mathrm{dl}$ (values after blood transfusion are accepted), levels of aspartate aminotransferase and alanine aminotransferase $\leq 100 \mathrm{IU} / \mathrm{l}$, total bilirubin level $\leq 1.5 \mathrm{mg} / \mathrm{dl}$, serum creatinine level $\leq 1.2 \mathrm{mg} / \mathrm{dl}$, creatinine clearance $\geq 60 \mathrm{ml} /$ min, normal electrocardiogram or electrocardiographic changes not requiring treatment]; and ix) written informed consent for voluntary participation in this study.

Exclusion criteria. The exclusion criteria were as follows: i) distinct evidence of infectious disease; ii) serious concurrent disease (cardiac disease, uncontrolled diabetes mellitus, malignant hypertension, bleeding tendency, etc.); iii) active double cancer; iv) interstitial pneumonia or pulmonary fibrosis; v) body fluid retention requiring treatment; vi) unstable angina, a history of myocardial infarction within 6 months before enrollment, or serious arrhythmias requiring treatment; vii) contraindications for irinotecan or nedaplatin; viii) diarrhea (watery stool); ix) intestinal paralysis or ileus; $x$ ) pregnant women, breastfeeding women, or women who want to become pregnant; xi) a history of serious drug hypersensitivity or drug allergy; and xii) patients considered unsuitable as subjects by their attending physicians for study-related reasons.

Treatment. Patients received irinotecan $\left(60 \mathrm{mg} / \mathrm{m}^{2}\right)$ on days 1 and 8 and nedaplatin $\left(80 \mathrm{mg} / \mathrm{m}^{2}\right)$ on day 1 of a 21-day cycle. After 1-3 courses of chemotherapy, extended hysterectomy was performed. If a partial or better response was obtained after 1 or 2 courses, or if a response was considered unlikely, surgery could be performed.

Criteria for skipping treatment with irinotecan on day 8. Laboratory tests were always performed 1 day before or on the same day as treatment with irinotecan on day 8 to confirm the severity of adverse drug reactions and the patients' status. Treatment with irinotecan on day 8 was skipped if patients met any of the following criteria: neutrophil count $<1500 / \mu 1$, platelet count $<100 \times 10^{3} / \mu 1$, grade 1 or higher infection, a fever of $38^{\circ} \mathrm{C}$ or higher, or grade 1 or higher diarrhea.

Criteria for starting the next course. Before starting the second and subsequent course of chemotherapy, laboratory tests were always performed within $24 \mathrm{~h}$ before the time scheduled for treatment to adequately confirm the severity of adverse drug reactions and the patients' status. The next course of treatment was postponed if patients did not meet the following criteria: neutrophil count $\geq 1500 / \mu 1$, platelet count $\geq 100 \times 10^{3} / \mu 1$, serum creatinine level $\leq 1.5 \mathrm{mg} / \mathrm{dl}$, grade 0 infection, grade 0 fever, and grade 0 diarrhea. However, the study treatment was discontinued if these criteria were not met up to a maximum of 5 weeks after the start of the previous course. Furthermore, if a granulocyte colony-stimulating factor (G-CSF) preparation was used to treat neutropenia, patients were observed for at least 3 days after the completion of treatment to confirm that the neutrophil count was $\geq 1500 / \mu 1$.

The dose for the next course of treatment was reduced according to the severity of the adverse drug reactions that occurred during the previous course. If grade 4 non-hematologic toxicity developed, the protocol treatment was discontinued.

Criteria for reduction of irinotecan dose. The dose of irinotecan was decreased $10 \mathrm{mg} / \mathrm{m}^{2}$ for the next course of treatment in patients who had any of the following conditions during the previous course: grade 3 or higher febrile neutropenia; grade 4 
neutropenia persisting for $\geq 5$ days; grade 3 or higher nonhematologic toxicity, excluding hair loss, nausea, vomiting, and fatigue; or if dose reduction was considered necessary by the patient's attending physician. Once the dose was reduced, treatment was continued at the lower dose (Table II).

Criteria for reduction of nedaplatin dose. The criteria for reducing the dose of nedaplatin were based on the platelet count. The dose of nedaplatin was reduced $10 \mathrm{mg} / \mathrm{m}^{2}$ for the next course in patients who had any of the following conditions during the previous course: grade 4 thrombocytopenia (platelet count, $<25 \times 10^{3} / \mu 1$ ); a bleeding tendency caused by grade 3 thrombocytopenia (platelet count, $\geq 25 \times 10^{3} / \mu 1$ to $<50 \times 10^{3} / \mu 1$ ); platelet transfusion was received; or if dose reduction was considered necessary by the patient's attending physician. Once the dose was reduced, treatment was continued at the lower dose.

Evaluation of adverse events. Adverse events were evaluated according to the JCOG Japanese version of the Common Terminology Criteria for Adverse Events (CTCAE), Version 3.0.

Surgery. Surgery was to be performed between 4 and 6 weeks after the completion of preoperative chemotherapy. In principle, radical hysterectomy was performed. However, if surgery was not considered feasible after NAC or if surgery was precluded by concurrent disease or other factors, non-surgical treatment such as radiotherapy could be administered at the discretion of the attending physician.

Postoperative treatment. In this clinical trial, postoperative therapy was not specified. Patients were followed up and given radiography or chemotherapy according to the criteria of each hospital.

Endpoints. The primary endpoint in this study was the response $(\mathrm{PR}+\mathrm{CR})$ rate. Response was evaluated at a single timepoint, referring to the World Health Organization Response Evaluation Criteria in Solid Tumors (RECIST guidelines). Time to treatment failure was not required. Secondary endpoints were: i) the number of courses required for response; ii) incidence of adverse events; iii) tumor marker levels (serum squamous cell carcinoma antigen); iv) completeness of surgery (presence or absence of residual tumor on intraoperative examination, rate of complete lymph node dissection, and negative resection margin rate); v) results of pathological examination of resected organs (histologic response, parametrial invasion, resection margin status, stromal invasion, vascular invasion, and lymphatic invasion); vi) relative ease or difficulty of surgery (bleeding volume and operation time); vii) proportion of patients with surgical complications; and viii) recurrence-free survival rate at 2 years.

Evaluation criteria for target lesion response. Tumor response was evaluated in accordance with RECIST guidelines. Before treatment and at the completion of each course, the longest diameter of target lesions was measured in a single direction on MRI or by direct measurement. We used MRI instead of computed tomography (CT) for two reasons. First, as compared with CT, MRI can more clearly depict tumor borders, facilitating the measurement of tumor diameter. Second, because cervical cancer invasion often extends vertically, MRI is more useful for
Table I. Patient characteristics.

Characteristics

Patients $(n=66)$

$\begin{array}{lr}\text { Age } & \\ \text { Median } & 47 \\ \text { Range } & 22-7 \\ \text { PS } & \\ 0 & 61 \\ 1 & 5 \\ \text { FIGO stage } & \\ \text { Ib2 } & 18 \\ \text { IIa } & 10 \\ \text { IIb } & 38\end{array}$

Tumor size $(\mathrm{cm})$

$\leq 4$

16

$>4$

50

measuring vertical extension on sagittal sections. Response was defined as: complete response (CR), disappearance of all target lesions including tumor-induced secondary changes; partial response (PR), $\mathrm{a} \geq 30 \%$ decrease in the sum of the longest diameter of target lesions, as compared with the value before the start of treatment; stable disease (SD): no evidence of tumor shrinkage corresponding to PR or of tumor growth corresponding to progressive disease; progressive disease (PD), a $\geq 20 \%$ increase in the sum of the longest diameter of target lesions, as compared with the smallest previous value; not evaluable (NE), examination cannot be performed for some reason or response is not evaluated to be CR, PR, PD, or SD.

Statistical analysis. Kaplan-Meier curves were generated for time to first recurrence and progression-free survival. We compared curves for the two groups with the log-rank test. All time estimates were done with the date of first chemotherapy as the baseline.

\section{Results}

A total of 68 patients were enrolled. Two patients did not meet the eligibility criteria and were excluded, and the remaining 66 were included in the full analysis set. As for their demographic characteristics, the median age was 47 years (range 22-71); the FIGO stage was Ib2 in 18 patients, IIa in 10, and IIb in 38; performance status was 0 in 61 patients and 1 in 5; and tumor diameter was $\leq 4 \mathrm{~cm}$ in 16 patients and $>4 \mathrm{~cm}$ in 50 (Table I). Radical hysterectomy was performed after NAC, thereby completing the protocol treatment, in 63 patients $(95.5 \%)$. Three patients discontinued protocol treatment: 1 directly refused treatment; 1 only received an exploratory laparotomy; and 1 did not undergo surgery because her attending physician preoperatively judged that operation was not feasible. The number of administered courses of NAC was 1 in 13 patients, 2 in 43, and 3 in 10. The mean interval from the date of staring course 1 of NAC to the date of starting the course 2 was 27.6 days (range 20-42). G-CSF was used in 10 of 53 
Table II. Clinical response of neoadjuvant chemotherapy with CPT-11+NED.

\begin{tabular}{lcrll}
\hline Clinical response & Patients & Response rate & \\
\hline CR & 2 & $3.0 \%$ & \\
PR & 48 & $72.7 \%$ & $75.8 \%$ \\
SD & 12 & $18.1 \%$ & & \\
PD & 0 & $0 \%$ & \\
Evaluation failure & 4 & $6.1 \%$ & \\
\hline
\end{tabular}

\begin{tabular}{|c|c|c|}
\hline $\begin{array}{l}\text { No. of courses required until } \\
\text { response }\end{array}$ & Average & \\
\hline 1 course & 30 & \\
\hline 2 courses & 19 & 1.42 courses \\
\hline 3 courses & 1 & \\
\hline
\end{tabular}

$\mathrm{CR}$, complete response; PR, partial response; $\mathrm{SD}$, stable disease; $\mathrm{PD}$, progressive disease.

patients. The reasons for postponing treatment were neutropenia in 35 patients, thrombocytopenia in 2, diarrhea in 1, and others in 5 . The mean interval from the date of staring the course 2 of NAC to the date of starting course 3 was 27.1 days (range 21-34). G-CSF was used in 2 of 10 patients. The reasons for postponing treatment were neutropenia in 7 patients and thrombocytopenia in 1 . Treatment with irinotecan was skipped on day 8 in $4(6.1 \%)$ of 66 patients during course 1, 10 (18.9\%) of 53 patients during course 2 , and $3(30.0 \%)$ of 10 patients during course 3 . All of these patients skipped treatment because of neutropenia. The dose of irinotecan in the next course was decreased from $60-50 \mathrm{mg} / \mathrm{m}^{2}$ in $4(6.1 \%)$ of the 66 patients. The reason for this dose reduction was grade 4 neutropenia in $2(50 \%)$ of the 4 patients. The dose of nedaplatin was reduced in $2(3 \%)$ of the 66 patients. In 1 patient the dose was decreased from 80 to $70 \mathrm{mg} / \mathrm{m}^{2}$, and in the other the dose was decreased to $60 \mathrm{mg} / \mathrm{m}^{2}$. The reasons for dose reduction were grade 3 and 4 thrombocytopenia.

The response rate, the primary endpoint of this study, was $75.8 \%$ (CR in 2 patients, $\mathrm{PR}$ in $48, \mathrm{SD}$ in 12 , PD in 0 , and $\mathrm{NE}$ in 4). The mean number of treatment courses required for a response was 1.42 ( 1 course in 30 patients, 2 courses in 19, and 3 courses in 1) (Table II). The combination of irinotecan and nedaplatin was considered an effective regimen for cervical cancer (squamous cell carcinoma).

The incidences of grade 3 or 4 hematological toxicities were as follows (in descending order): neutropenia $72.2 \%$, leukopenia $16.7 \%$, anemia $13.6 \%$, thrombocytopenia $7.6 \%$, febrile neutropenia $1.5 \%$, and elevations of alanine aminotransferase and aspartate aminotransferase $1.5 \%$ (Table III). The incidences of grade 3 or 4 non-hematologic toxicities were: diarrhea $6.1 \%$, nausea $3 \%$, anorexia $1.5 \%$, vomiting $1.5 \%$, fever $1.5 \%$, allergic reactions $1.5 \%$, ileus $1.5 \%$, and vesicovaginal fistula $1.5 \%$ (Table IV). There were no deaths or other serious adverse events.

The serum level of squamous cell carcinoma antigen, a tumor marker, was abnormal $(\geq 1.5 \mathrm{ng} / \mathrm{ml})$ before treatment in $52(78.8 \%)$ of 66 patients. After chemotherapy, the level fell to the normal range in $29(55.8 \%)$ of these patients before surgery.
Table III. Hematological toxicities of chemothrapy with CPT-11+NED.

\begin{tabular}{lrrrrrr}
\hline & \multicolumn{6}{c}{ Grade } \\
\cline { 2 - 7 } Toxicities & 0 & 1 & 2 & 3 & 4 & G3/4 (\%) \\
\hline Leukopenia & 11 & 10 & 34 & 8 & 3 & 16.7 \\
Neutropenia & 7 & 4 & 8 & 37 & 10 & 71.2 \\
Febrile neutoropenia & 65 & 0 & 0 & 1 & 0 & 1.5 \\
Anemia & 15 & 15 & 27 & 8 & 1 & 13.6 \\
Thrombocytropenia & 34 & 20 & 7 & 2 & 3 & 7.6 \\
AST/GOT evelation & 53 & 12 & 1 & 0 & 0 & 0 \\
ALT/GPT evelation & 47 & 14 & 4 & 1 & 0 & 1.5 \\
Creatinine & 65 & 1 & 0 & 0 & 0 & 0 \\
Bilirubine & 60 & 4 & 2 & 0 & 0 & 0 \\
Hypoalbuminemia & 61 & 3 & 2 & 0 & 0 & 0 \\
\hline
\end{tabular}

NCI-CTCAE ver.3.0.

Table IV. Non-hematologic toxicities of chemothrapy with CPT-11+NED.

\begin{tabular}{lrrrrrr}
\hline & \multicolumn{6}{c}{ Grade } \\
\cline { 2 - 7 } Toxicities & 0 & 1 & 2 & 3 & 4 & G3/4 (\%) \\
\hline Anorexia & 14 & 38 & 13 & 1 & 0 & 1.5 \\
Nausea & 8 & 41 & 15 & 2 & 0 & 3.0 \\
Vomiting & 31 & 22 & 12 & 1 & 0 & 1.5 \\
Diarrhea & 26 & 24 & 12 & 4 & 0 & 6.1 \\
Fever & 46 & 14 & 5 & 1 & 0 & 1.5 \\
Hemorrhage & 61 & 5 & 0 & 0 & 0 & 0 \\
Hair loss & 11 & 37 & 18 & 0 & 0 & 0 \\
Allergic reaction & 62 & 3 & 0 & 1 & 0 & 1.5 \\
Edema & 60 & 4 & 2 & 0 & 0 & 0 \\
Ileus & 63 & 0 & 2 & 1 & 0 & 1.5 \\
Constipation & 54 & 10 & 2 & 0 & 0 & 0 \\
Pyelonephritis & 63 & 1 & 2 & 0 & 0 & 0 \\
Fatigue & 64 & 2 & 0 & 0 & 0 & 0 \\
Weight loss & 43 & 19 & 4 & 0 & 0 & 0 \\
Fistula (GU-bladder & 65 & 0 & 0 & 1 & 0 & 1.5 \\
vagina) & & & & & & \\
\hline
\end{tabular}

NCI-CTCAE ver.3.0.

Among the 50 patients who responded to NAC (CR+PR), the squamous cell carcinoma antigen level was abnormal in 41 $(82.0 \%)$ before treatment. In $25(61.0 \%)$ of these patients, the level decreased to normal after chemotherapy.

As for the radicality of surgery, $4(6.4 \%)$ of 63 patients were judged by the operator to have residual tumor or to have undergone incomplete lymph node resection, and 7 (11.1\%) had positive resection margins on histopathological examination of their resected specimens. 
Table V. Pathological examination.

\begin{tabular}{llr}
\hline Pathological findings & \multicolumn{1}{c}{ Yes } & No \\
\hline Parametrial involvement & 15 & 48 \\
Histologic margin & 7 & 56 \\
Depth of stromal invasion & $21(<50 \%)$ & 6 \\
& $36(>50 \%)$ & \\
Vascular-lymphatic involvement & 31 & 32 \\
Lymph node metastasis & 19 & 44 \\
\hline
\end{tabular}

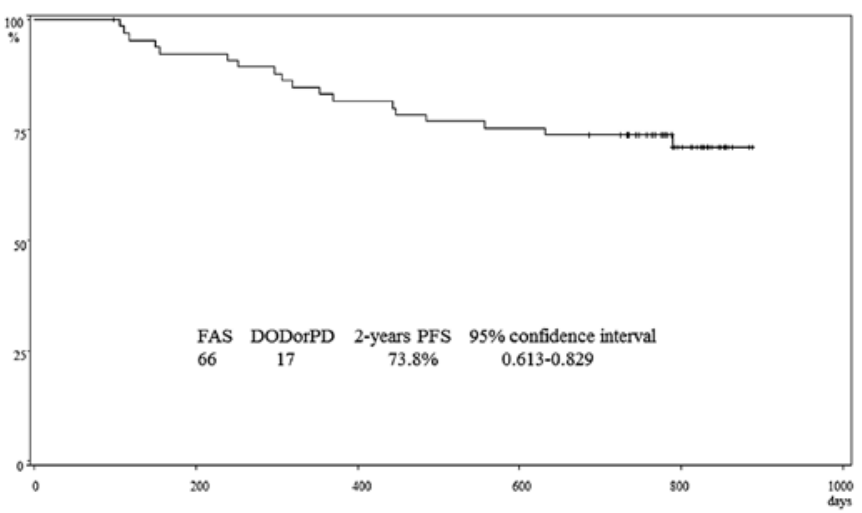

Figure 1. The two-year progression-free survival of all patients Kaplan-Meier method)

Overall, 57 (90.5\%) of 63 patients had a histologic response on histopathological evaluation of their resected specimens (grade 0 in 6 patients, grade $1 \mathrm{a}$ in 23 , grade $1 \mathrm{~b}$ in 8 , grade 2 in 22 , and grade 3 in 4). Among the 63 patients, parametrial invasion was present in 15 (23.8\%), stromal invasion in 57 (90.5\%; $<50 \%$ in 21 and $\geq 50 \%$ in 36 ), vascular invasion in $31(49.2 \%)$, and lymph node metastasis in 19 (30.2\%) (Table V). Given that $38(57.6 \%)$ of the 66 patients had stage IIb disease, the improvement in histopathological findings after NAC was regarded to be considerable.

The mean bleeding volume was $998 \mathrm{ml}$ (range 158-3362), and the mean operation time was 294 min (range 136-566). As for surgical complications, grade 3 or 4 intraoperative bleeding occurred in $3.2 \%$ of the patients, which did not differ from the incidence associated with conventional extended hysterectomy. Dysuria was grade 0 in $33.9 \%$ of the patients, grade 1 in $25.8 \%$, grade 2 in $19.4 \%$, and grade 3 in $21.0 \%$. The incidence of grade 1 or 2 lymphatic cyst was somewhat high (27.4\%).

Two years after surgery, 17 patients had recurrence, including 9 who died of cancer. The progression-free survival rate at 2 years was $73.8 \%$ (95\% confidence interval, $0.613-0.829$ ) (Fig. 1). In this study, additional postoperative treatment was not specified and was left to the treatment policy of each hospital and the discretion of the attending physician. The breakdown of postoperative therapy was: no additional treatment in 30 patients (recurrence in 8), radiotherapy in 5 (recurrence in 1), concurrent chemoradiotherapy in 15 (recurrence in 7), and chemotherapy in 15 (recurrence in 1). In patients with target lesions only in the cervix and those with target lesions in both the cervix and lymph

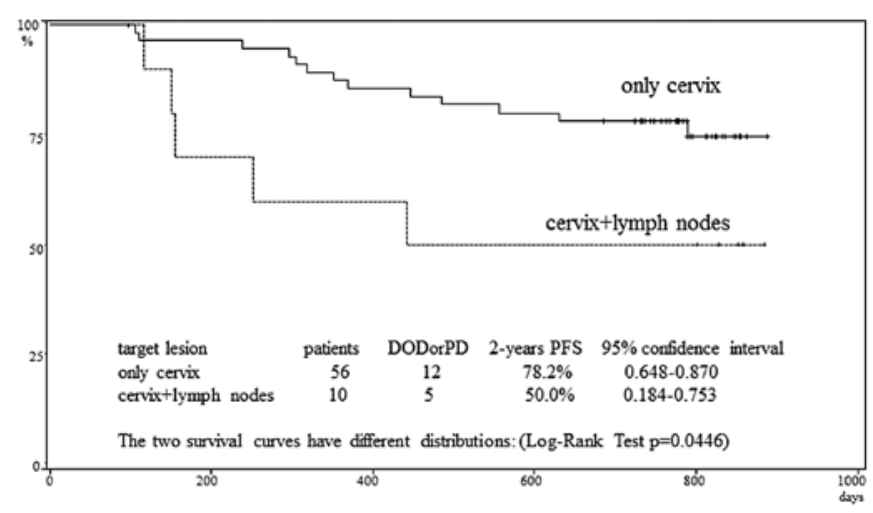

Figure 2. The two-year progression-free survival according to target lesion; only cervix vs. cervix+lymph nodes (Kaplan-Meier method).

nodes, the progression-free survival rate at 2 years was 78.2 and $50.0 \%$, respectively. This difference was significant $(\mathrm{p}=0.0446)$ (Fig. 2). The progression-free survival rate at 2 years according to disease stage was $94.1 \%$ for Ib2, $60.0 \%$ for IIa, and $68.4 \%$ for IIb. These differences were not significant.

\section{Discussion}

The response rate, the primary endpoint, was $75.8 \%$ (CR in 2 patients, $\mathrm{PR}$ in 48, SD in 12, PD in 0 , and NE in 4). The tumor shrinkage rate was $<30 \%$ in 11 of the 12 patients with SD. The response rate was non-inferior to those previously reported for NAC in patients with cervical cancer. It is noteworthy that only 1.42 courses of NAC on average were required to produce a response ( 1 course in 30 patients, 2 courses in 19, and 3 courses in 1). Fifty-six of 66 patients $(84.8 \%)$ received 1 or 2 courses of NAC. Moreover, no patient had PD. CPT-11 and NED had a high response rate against primary lesions, as well as a prompt onset of effect, making it an optimal regimen for NAC.

Although a number of studies of NAC using a variety chemotherapeutic agents have been evaluated in patients with locally advanced cervical cancer and high response rates ranging from 76 to $95 \%$ have been demonstrated (12-17).

The rate of progression-free survival at 2 years was $73.8 \%$ (95\% confidence interval, 0.613-0.829) in initially treated patients with stage Ib2 to IIb cervical squamous cell carcinoma who received NAC with irinotecan plus nedaplatin followed by radical hysterectomy. Because $57.6 \%$ of the study group had stage IIb disease and $75.8 \%$ had tumors exceeding $4 \mathrm{~cm}$ in diameter, we consider our results to be satisfactory. In addition, the study protocol did not specify additional treatment after surgery, and such treatment was left to the treatment policies of each hospital and the discretion of the attending physician. Postoperative treatment was performed as follows: no additional treatment in 30 patients (recurrence in 8), radiotherapy in 5 (recurrence in 1), concurrent chemoradiotherapy in 15 (recurrence in 7), and chemotherapy in 15 (recurrence in 1). Because chemotherapy was associated with good outcomes, future studies should assess whether multidisciplinary treatment combining surgery with preoperative and postoperative chemotherapy can improve outcomes $(7,18)$. Patients with lymph nodes as target lesions had significantly poorer outcomes. More 
aggressive, individualized treatment strategies may be necessary in this subgroup of patients.

In Japan, stage Ib2 to IIb cervical cancer is generally treated by radical hysterectomy. In the United States, the National Comprehensive Cancer Network (NCCN) and National Cancer Institute (NCI) guidelines recommend concurrent chemoradiotherapy for patients with stage Ib2 to IIb disease, and surgery is not included as a treatment option $(19,20)$. Since the 1980 s, however, many attempts have been made to improve survival by performing surgery after preoperative chemotherapy in patients with stage Ib2 to IIb cervical cancer (21-28). Preoperative chemotherapy may eliminate micrometastases, facilitate complete tumor resection, and enable resection of previously unresectable tumors.

Some studies have shown that NAC is therapeutically useful, whereas others have not. The Meta-analysis Group in the UK conducted a meta-analysis of 6 randomized controlled trials comparing NAC plus surgery with surgery alone. NAC was found to significantly improve progression-free survival (hazard ratio, $0.76 ; 95 \%$ confidence interval $0.62-0.94 ; p=0.01$ ), but not overall survival (hazard ratio, 0.85; 95\% confidence interval 0.67-1.07; $\mathrm{p}=0.17)$. In the NAC group, histopathological findings such as lymph node metastasis and parametrial invasion improved significantly. The NAC group also showed slight trends toward better outcomes in terms of local recurrence, distant recurrence, and resection rate. However, the meta-analysis concluded that it was unclear whether NAC improves long-term outcomes (29).

In 2003, the results of a meta-analysis of 5 clinical studies comparing NAC plus surgery with surgery alone in 872 patients with stage I or II (some with stage III) cervical cancer were reported. As compared with surgery alone, NAC plus surgery was found to significantly improve overall survival (hazard ratio, 0.65) and disease-free survival (hazard ratio, 0.68) at 5 years (30). However, this meta-analysis had several limitations, such as the inclusion of patients with various stages of cervical cancer and the lack of a comparison with chemoradiotherapy. Preoperative chemotherapy has thus not been accepted as standard treatment.

Since 2002, the European Organization for Research and Treatment of Cancer (EORTC) has been conducting a randomized controlled trial (EORTC 55994) comparing concurrent chemoradiotherapy, currently standard treatment, with preoperative chemotherapy followed by surgery in patients with stage Ib2-IIb cervical cancer. This study is ongoing and conclusions have yet to be reached (30). At present, evidence demonstrating that NAC plus surgery is superior to surgery alone or concurrent chemoradiotherapy is still not available (31).

\section{Acknowledgements}

We are indebted to the following participating hospitals for cooperating in patient enrollment: Hyogo Cancer Center, Tohoku University Hospital, St. Marianna University School of Medicine Hospital, Iwate University School of Medicine Hospital, Tottori University Hospital, Kitasato University, Osaka City General Hospital, Cancer Institute Hospital Ariake, National Hospital Organization Kure Medical Center-Chugoku Cancer Center, Jikei University Kashiwa Hospital, Keio University Hospital, Nagasaki University Hospital of Medicine and Dentistry, Hamamatsu Medical Center, Shinshu University Hospital,
Hiroshima University Hospital, Dokkyo Medical University Hospital, National Hospital Organization Shikoku Cancer Center, Kochi Health Sciences Center, Yamaguchi University Hospital, Kinki University Hospital, Nara Medical University Hospital, Jikei University Hospital, Tokyo Dental College Ichikawa General Hospital, Hirosaki University Hospital, National Hospital Organization Fukuyama Medical Center, Kansai Rosai Hospital, Fukuoka University Hospital, Sapporo Railway Hospital, Wakayama Medical University Hospital, Tokyo Medical University Hospital, Kumamoto University Hospital, Japanese Red Cross Ashikaga Hospital, and Nagasaki Municipal Hospital. We thank all members of JGOG.

\section{References}

1. Yamamoto K, Kokawa K, Umesaki N, et al: Phase I study of combination chemotherapy with irinotecan hydrochloride and nedaplatin for cervical squamous cell carcinoma: Japanese Gynecologic Oncology Group study. Oncol Rep 21: 1005-1009, 2009.

2. Takeuchi S, Dobashi K, Fujimoto S, et al: A late phase II study of CPT-11 on uterine cervical cancer and ovarian cancer. Jpn J Cancer Chemother 18: 1681-1689, 1991.

3. Kanazawa F, Koizumi F, Koh Y, et al: In vitro synergistic interactions between the cisplatin analogue nedaplatin and the DNA topoisomerase I inhibitor irinotecan and the mechanism of this interaction. Clin Cancer Res 7: 202-209, 2001.

4. Sugiyama T, Yakushiji M, Noda K, et al: Phase II study of irinotecan and cisplatin as first-line chemotherapy in advanced or recurrent cervical cancer. Oncology 58: 31-37, 2000.

5. Sugiyama T, Nishida T, Kumagai S, et al: Combination therapy with irinotecan and cisplatin as neoadjuvant chemotherapy in locally advanced cervical cancer. Br J Cancer 81: 95-98, 1999.

6. Raspagliesi F, Ditto A, Selvaggi L, et al: A phase 2 multicenter study of irinotecan and cisplatinum as neoadjuvant treatment in patients with locally advanced cervical cancer. Int J Gynecol Cancer 20: 1569-1575, 2010.

7. Matsumura M, Takeshima N, Ota T, et al: Neoadjuvant chemotherapy followed by radical hysterectomy plus postoperative chemotherapy but no radiotherapy for Stage IB2-IIB cervical cancer - irinotecan and platinum chemotherapy. Gynecol Oncol 119: 212-216, 2010

8. Kato T, Nishimura H, Yakushiji M, et al: Phase II study of 254-S (cis-diammine glycolate platinum) for gynecological cancer. Jpn J Cancer Chemother 19: 695-701, 1992.

9. Machida S, Ohwada M, Fujiwara H, et al: Phase I study of combination chemotherapy using irinotecan hydrochloride and nedaplatin for advanced or recurrent cervical cancer. Oncology 65: 102-107, 2003.

10. Tsuda H, Hashiguchi Y, Nishimura S, et al: Phase I-II study of irinotecan plus nedaplatin with recombinant human granulocyte colony-stimulating factor support in patients with advanced or recurrent cervical cancer. Br J Cancer 91: 1032-1037, 2004.

11. Ohwada M, Machida S, Fujiwara H, et al: Phase II study of combination chemotherapy using irinotecan and nedaplatin for patients with primary advanced or recurrent cervical cancer. Proc ASCO: J Clin Oncol 22 (Suppl 14): abs. 5088, 2004.

12. Zanetta G, Fei F, Mangioni C, et al: Chemotherapy with paclitaxel, ifosmide, and cisplatin for the treatment of squamous cell cervical cancer. Semin Oncol 27: 23-27, 2000.

13. Haung HJ, Chang TC, Hong JH, et al: Prognostic value of age and histologic type in neoadjuvant chemotherapy plus radical surgery for bulky $(\geq 4 \mathrm{~cm})$ stage IB and IIA cervical carcinoma. Int J Gynecol Cancer 13: 204-221, 2003.

14. D'Agostino DG, Distefano M, Greggi S, et al: Neoadjuvant treatment of locally advanced carcinoma of the uterine cervix with epirubicin, paclitaxel and cisplatin. Cancer Chemother Pharmacol 49: 256-260, 2002

15. Vagno GD, Cormio G, Pinata S, et al: Cisplatin and vinorelbine as neoadjuvant chemotherapy in locally advanced cervical cancer: a phase II study. Int J Gynecol Cancer 13: 308-312, 2003.

16. Duenas-Gonzales A, Lopez-Graniel C, Gonzalez-Enciso A, et al: A phase II study of multimodality treatment for locally advanced cervical cancer: neoadjuvant carboplatin and paclitaxel followed by radical hysterectomy and adjyvant cisplatin chemoradiation. Ann Oncol 14: 1278-1284, 2003. 
17. Umesaki N, Fujii T, Nishimura R, et al: Combination chemotherapy with iirunotecan (irinotecan) and mitomycin C (MMC) for advanced or recurrent squamous cell carcinoma of the cervix: Japanese Gynecologic Oncology Group (JGOG) study. Proc Am Soc Clin Oncol 22: 465, abs. 1869, 2003.

18. Takeshima N, Umayahara K, Fujiwara K, et al: Treatment results of adjuvant chemotherapy after radical hysterectomy for intermediate-and high risk stage IB-IIA cervical cancer. Gynecol Oncol 103: 618-622, 2006.

19. NCCN Clinical Practice Guidelines in Oncology-Cervical Cancer-v2. National Comprehensive Cancer Network, 2006.

20. Cervical Cancer Treatment $\left(\mathrm{PDQ}^{\circledR}\right)$, Health Professional Version National Cancer Institute in the United States (web-site), 2011.

21. Sardi JE, Sananes CE, Giaroli AA, et al: Long-term follow-up of the first randomized trial using neoadjuvant chemotherapy in stage Ib squamous carcinoma of the cervix: the final results. Gynecol Oncol 67: 61-69, 1997.

22. Benedetti-Panici P, Greggi S, Scambia G, et al: Long-term survival following neoadjuvant chemotherapy and radical surgery in locally advanced cervical cancer. Eur J Cancer 34: 341-346, 1998.

23. Chang TC, Lai CH, Hong JH, et al: Randomized trial of neoadjuvant cisplatin, vincristine, bleomycin, and radical hysterectomy versus radiation therapy for bulky stage IB and IIA cervical cancer. J Clin Oncol 18: 1740-1747, 2000.

24. Buda A, Fossati R, Colombo N, et al: Randomized trial of neoadjuvant chemotherapy comparing paclitaxel, ifosfamide, and cisplatin with ifosfamide and cisplatin followed by radical surgery in patients with locally advanced squamous cell cervical carcinoma: the SNAP01 (studio neo-adjuvante portio) Italian Collaborative Study. J Clin Oncol 23: 4137-4145, 2005.
25. Tzioras S, Pavlidis N, Paraskevaidis E, et al: Effects of different chemotherapy regimens on survival for advanced cervical cancer: systematic review and meta-analysis. Cancer Treat Rev 33: 24-38, 2007.

26. Katsumata N, Yoshikawa H, Hirakawa T, et al: Phase III randomized trial of neoadjuvant chemotherapy (NAC) followed by radical hysterectomy (RH) versus RH for bulky stage I/II in cervical cancer (JCOG0102). Proc Am Soc Clin Oncol 24 (S18): 1, abs. 5013, 2006.

27. Eddy GL, Bundy BN, Creasman WT, et al: Treatment of (bulky) stage IB cervical cancer with or without neoadjuvant vincristine and cisplatin prior to radical hysterectomy and pelvic/para-aortic lymphadenectomy: a phase III trial of the Gynecologic Oncology Group. Gynecol Oncol 106: 362-369, 2007.

28. Mossa B, Mossa S, Corosu L, et al: Follow-up in a long-term randomized trial with neoadjuvant chemotherapy for squamous cell cervical carcinoma. Eur J Gynecol Oncol 31: 497-503, 2010.

29. Rydzewska L, Tierney J, Vale CL, et al: Neoadjuvant chemotherapy plus surgery versus surgery for cervical cancer. Cochrane Database Syst Rev 20 (1): CD007406, 2010.

30. Tierney J: Neoadjuvant chemotherapy for locally advanced cervical cancer: a systematic review and meta-analysis of individual patient data from 21 randomised trials. Eur J Cancer 39: 2470-2086, 2003.

31. Gonzalez-Martin A, Gonzalez-Cortijo L, Carballo N, et al: The current role of neoadjuvant chemotherapy in the management of cervical carcinoma. Gynecol Oncol 110: S36-S40, 2008. 